\title{
INVESTIGACIONES
}

\section{Participación y expectativas de los padres sobre la educación de sus hijos en una escuela pública}

\author{
Involvement and expectations of parents regarding the education \\ of their children in a public school
}

\author{
Alejandro Sánchez Oñate, ${ }^{a}$ Fernando Reyes Reyes, ${ }^{b}$ \\ Verónica Villarroel Henríquez $z^{b c}$ \\ ${ }^{a}$ Universidad de Concepción \\ Telf.: (56) 9 99382815. Correo electrónico: asanchezo@udec.cl \\ ${ }^{\mathrm{b}}$ Universidad del Desarrollo \\ Telf.: (56) 998184325 . Correo electrónico: vvillarroel@udd.cl \\ ‘Telf.: (56) 9 94899286. Correo electrónico: freyes@udd.cl
}

\begin{abstract}
RESUMEN
Este estudio indagó la relación entre la participación y las expectativas de los apoderados sobre la educación escolar de sus hijos en el contexto de una escuela pública del sur de Chile. La investigación demuestra que estas variables son claves en el proceso educativo de los niños, existiendo evidencias de una directa relación con los resultados de aprendizaje. Se utilizó un diseño mixto secuencial-explicativo. En una primera fase cuantitativa se realizó un análisis de regresión lineal múltiple, arrojando un modelo predictivo del rendimiento académico que agrupa a las variables nivel socioeconómico, participación y expectativas educativas. En la segunda fase de tipo cualitativa, los resultados muestran distintas condiciones intervinientes en la dinámica, destacando las barreras socioeconómicas como mediadoras de las expectativas y la participación de los padres; ampliando la discusión acerca del rol de los establecimientos en la promoción de la participación de los apoderados en la educación escolar.
\end{abstract}

Palabras clave: participación de los apoderados, expectativas educativas, rendimiento académico, educación pública, investigación educativa.

\begin{abstract}
This study investigated the relationship between parents involvement and expectations on school education of their children, in the context of a public school in southern Chile. Educational research shows that these variables are key in the process of educating children, there is evidence of a direct relationship with the learning outcomes. A sequential-explanatory mixed design was used. In a first quantitative phase, analysis of multiple linear regression was performed, yielding a predictive model of academic performance that brings together the socioeconomic variables, participation and educational expectations. In the second phase of qualitative type, the results allow different dynamics involved conditions, highlighting the socioeconomic barriers as mediators expectations and parental involvement; allowing broader discussion about the role of institutions in promoting the participation of parents in school education.
\end{abstract}

Key words: parent involvement, educational expectations, academic achievement, public education, educational research. 


\section{INTRODUCCIÓN}

La educación chilena ha sido objeto de discusión durante las últimas décadas, tanto a nivel nacional como internacional, al constituirse como ejemplo de un sistema educativo caracterizado por una lógica de mercado asociada a la competencia y la privatización de la educación, que sostiene y posibilita una alta segregación social de los establecimientos educativos. Por lo tanto, al abordar una temática educativa compleja como la participación y las expectativas de los padres sobre la educación de sus hijos en el contexto de un establecimiento público, es menester adentrarse en la comprensión del marco político y social en el que este se inserta; especialmente si consideramos que una de las consecuencias de la instauración de este sistema educativo ha sido la crisis en la educación pública, expresada en el decrecimiento del sector municipal debido a la migración de los estudiantes hacia el sector privado y a los bajos resultados académicos de los estudiantes (Cornejo, 2006; Moreno-Doña y Gamboa, 2014).

De acuerdo con Vain (2009), la escuela nace en el contexto histórico de la modernidad, a partir de un pacto en el que las familias entregaron al Estado la responsabilidad de educar a sus hijos. Así, la escuela se transformaría en el motor que haría posible la inclusión y movilidad social de los estudiantes. Sin embargo, al imbricarse la educación dentro de un sistema social y económicamente capitalista — como el caso chileno-, se quebranta la promesa de la escuela pública que abre a los sectores marginados la posibilidad de insertarse en los estratos altos de la sociedad, ya que esta opción pasa a estar condicionada a las necesidades de expansión del mercado y se limita por la oferta de distintos niveles de calidad educativa según los sectores sociales atendidos. Este panorama socioeducativo permite explicar el hecho de que la demanda de la escuela por una mayor participación de los padres en la educación de sus hijos no encuentre la respuesta esperada.

Con todo, la educación sigue siendo para la mayoría de la población el principal vehículo de movilidad social, impactando en el futuro socioeconómico de las personas (Anabalón et al., 2008). Si bien existe evidencia amplia acerca de la influencia que ejercen las condiciones socioeconómicas de las familias sobre el desarrollo de los estudiantes, se ha evidenciado también que esa influencia puede ser revertida o atenuada por la acción que lleva a cabo una escuela (Bellei et al., 2014).

En definitiva, hoy en día se admite que la responsabilidad de educar está tanto en las familias como en los establecimientos educacionales. Tras los primeros años de educación en el hogar, las niñas y niños comienzan a desarrollar la autonomía y la independencia que facilitarán el inicio de las primeras relaciones sociales fuera de la familia. Es en este proceso donde la familia debe contribuir a generar las condiciones afectivas, temporales, valóricas, culturales y económicas que permitan un desarrollo integral de sus hijos e hijas en la sociedad. Luego, la escuela pasa a ser la unidad central donde tienen lugar los procesos de integración social y de socialización (Anabalón et al., 2008).

En este binomio de escuela y familia, el concepto de educabilidad cobra también importancia. Este se refiere al conjunto de recursos y predisposiciones que hacen posible que un niño o una niña pueda educarse, como también a las condiciones sociales que favorecen la adquisición de dichos recursos. Adicionalmente, se distingue entre educabilidad interna, referida a las condiciones derivadas de la escuela, tales como infraestructura, objetivos estratégicos, prácticas docentes, aspectos curriculares, entre otros; y educabilidad externa, que alude a las condiciones del contexto familiar y cultural del niño, tales como el barrio, 
el ingreso socioeconómico, las amistades, los bienes materiales, entre otros. Así, con una combinación adecuada de estos elementos mediada de forma indispensable por la participación de las familias en el proceso educativo de los estudiantes, se alcanzarían altos niveles de resultados educativos (Tedesco, 2010 Cit. en Anabalón et al., 2008).

\subsection{PARTICIPACIÓN DE LOS PADRES Y MADRES EN LA EDUCACIÓN}

El estudio de la participación de los padres (o de las familias) en la educación se ha abordado desde distintas conceptualizaciones, entre las cuales se pueden destacar:

a) Una visión de participación entendida como comunicación con la escuela y apoyo de los aprendizajes del aula (Valdés, Martín y Sánchez-Escobedo, 2009; Zhan, 2006), que incluiría aspectos como la asistencia a la escuela, la comunicación con los maestros, el conocimiento del funcionamiento de la escuela, la comunicación con los hijos y la ayuda en la realización de tareas.

b) Una perspectiva que añade a la visión anterior la dimensión de provisión de recursos educativos (Altschul, 2012), tales como la inversión de tiempo en instrucción fuera del aula y actividades que enriquecen los aprendizajes de los estudiantes.

c) Una perspectiva que abordaría la participación en un sentido democrático, ligado al grado en que los padres, madres y apoderados participan en la toma de decisiones dentro de un establecimiento (Bolívar, 2006; Gatt y Petreñas, 2012; Martínez y Niemelä, 2010; Ministerio de Educación, 2009; Valdés, Carlos y Arreola, 2013).

Así, dentro de las dos primeras conceptualizaciones de la participación de los padres en la educación, Altschul (2012) y Valdés et al. (2013) coinciden en señalar que correspondería a un constructo bidimensional, compuesto por la inversión de recursos en la educación (apoyo personal, provisión de materiales, enseñanza extracurricular) y por la participación de los padres en la educación de la escuela (conversar acerca de la escuela y los profesores, ayudar con las tareas escolares, formar parte de organizaciones escolares).

Respecto de la visión más amplia de participación, según Valdés et al. (2013), esta se enmarca dentro de un concepto denominado participación social. La participación social corresponde a un mecanismo que resalta el valor de la democracia y propende al involucramiento de todos los actores sociales en la búsqueda de beneficios comunes. En el ámbito de la escuela, la participación social correspondería al involucramiento activo de las autoridades de gobierno, empresarios, sociedad civil, directivos, profesores, familias y estudiantes en pos del mejoramiento de la calidad educativa.

En el título preliminar, párrafo uno, artículo tercero de la Ley General de Educación (Ministerio de Educación, 2009) se reconoce como un principio inspirador la participación, en la que los miembros de la comunidad educativa tienen derecho a ser informados y a participar en el proceso educativo, agregando en el artículo 15 el deber que tiene el establecimiento de promover esta participación a través de la formación de Centros de Alumnos, Centros de Padres y Apoderados, Consejos de Profesores y Consejos Escolares.

Este carácter democrático de la participación de los padres y madres en la educación de sus hijos estaría también asociado a la posibilidad de presionar a las escuelas para exigir una enseñanza de calidad, exigir información acerca de sus alumnos, enfrentar problemáticas de violencia escolar y, en definitiva, la posibilidad de demandar sus derechos (Valdés y Urías, 2010; Valdés et al., 2013). 
En esta misma línea, Gatt y Petreñas (2012) han conceptualizado cinco formas de participación de las familias dentro de un establecimiento, las cuales se complementarían para favorecer el impacto en los resultados de aprendizaje de los estudiantes, es decir, cada una de ellas no excluye a la otra.

Desde este modelo, la participación podría limitarse a la recepción de información sobre la gestión del establecimiento (participación informativa) o al carácter consultivo que tienen las familias a través de los centros de padres y apoderados (participación consultiva). Estas dos primeras formas tendrían un menor impacto positivo en los resultados de aprendizaje. Por otra parte, la posibilidad de participar directa y representativamente en los procesos de toma de decisiones, junto con la supervisión de la rendición de cuenta de los resultados de aprendizaje (participación decisiva), la evaluación de dichos resultados (participación evaluativa) y la participación en los procesos de enseñanza dentro y fuera de la escuela (participación educativa) favorecería un impacto positivo en los resultados de aprendizaje de los estudiantes (Gatt y Petreñas, 2012).

Respecto de la relación entre participación de las familias y resultados de aprendizaje, Valdés y Urías (2010) encontraron que la participación estaría asociada a mayores logros académicos, especialmente en lectura, siendo las habilidades y la motivación de los padres variables mediadoras en el aprendizaje y desarrollo cognitivo de los niños y niñas. Esta misma relación mediadora sería confirmada por Bolívar (2006), quien sostiene que la motivación, entendida como sentido de responsabilidad personal y/o compartida por los resultados de aprendizaje, junto con la percepción de eficacia de los padres, entendida como la creencia de que sus acciones personales pueden efectivamente ayudar al niño a aprender; favorecerían una mayor o menor implicación en la educación de sus hijos. Por último, se ha encontrado en estudios de meta-análisis que esta asociación estaría vinculada más bien a la participación de los padres y madres en las actividades que se desarrollan directamente en la escuela, no así a la participación de estos limitada al acompañamiento de las tareas educativas en el hogar (Zhan, 2006).

Por otra parte, entre los predictores de la participación de los padres y madres en la educación, se ha identificado al profesor como principal factor facilitador de esta, al ser un puente entre los apoderados y la escuela. También se ha encontrado que el haber pertenecido a la misma escuela de los hijos fortalecería la participación y el sentido de pertenencia con la comunidad escolar (Rivera y Milicic, 2006).

En relación con las barreras a la participación en la escuela, Anabalón et al. (2008) identificaron los compromisos económicos, las jornadas de trabajo extensas y el nivel educacional de los padres como los principales factores que limitan la participación de estos en la escuela. Por otra parte, Rivera y Milicic (2006) encontraron que se percibe más fácil participar en la educación básica que en la educación media, pues en la primera existen mayores necesidades de atención de los padres por parte de los niños, mientras que en la educación media se requiere de competencias parentales más complejas para tratar con los adolescentes.

Respecto de las consecuencias de la participación de los padres y madres en la educación, se pueden identificar positivamente el aumento del sentimiento de autoconfianza en la familia, la formación de una visión más positiva de la escuela y los profesores (Valdés y Urías, 2010), la disminución del efecto de factores contextuales tales como el bajo nivel socioeconómico o la baja escolaridad de los padres (Silas, 2008), aumento de interés en los hijos por participar en las actividades escolares y la mejora en las condiciones de infraestructura (Rivera y Milicic, 2006). 
En el estudio citado de Rivera y Milicic (2006), realizado dentro del contexto chileno, se encontró que existían tres estilos de participación de los padres y madres. El grupo más participativo correspondería a aquellos que voluntariamente están vinculados de forma permanente con la escuela, disfrutando de esa participación; el grupo menos participativo estaría compuesto por los apoderados que no asisten por razones de trabajo o desinterés, o que asisten ocasionalmente para manifestar algún reclamo; y un tercer grupo intermedio correspondería a aquellos que se limitan a asistir a las reuniones de apoderados, acostumbrándose a retirarse antes de tiempo.

Por otra parte, en el contexto de las escuelas municipales chilenas, de acuerdo con Román (2014), la participación y compromiso de padres y madres de estudiantes se ha reducido en los últimos años, lo que desde la perspectiva de estos actores se atribuye fundamentalmente a la realidad familiar (familias uniparentales correspondientes a madres trabajadoras) y en menor medida al desinterés de los padres por la educación de sus hijos, quedando así un número menor de apoderados que desarrolla un vínculo más estrecho y responsable con la escuela.

En otro estudio realizado en el contexto de familias en condición de pobreza en Chile (Anabalón et al., 2008), la participación en la escuela por parte de los padres respondería efectivamente a una aspiración de movilidad social a través de la educación, existiendo la creencia de la educación como legado familiar. En este mismo contexto, las barreras más comunes que se presentarían serían la desesperanza aprendida por el registro familiar de baja escolaridad, el embarazo adolescente, el consumo de drogas y alcohol, y la dualidad entre las expectativas de los padres y las de sus hijos, generando una sensación de incertidumbre frente al futuro.

\subsection{EXPECTATIVAS DE LOS PADRES SOBRE LA EDUCACIÓN}

De acuerdo con la literatura (Intxausti, Etxeberria y Joaristi, 2014; Stull, 2013), la expectativa se refiere a la esperanza de alcanzar cierto logro en la medida que se proporcionen las oportunidades deseadas para ello, o la creencia de lo que probablemente ocurrirá en el futuro. En relación con las expectativas educativas, se referirían al nivel educativo que realmente se cree alcanzar. En este sentido, las expectativas se distinguen del concepto de aspiración, que alude a aquellos deseos que exceden a lo que racionalmente se esperaría.

El estudio de las expectativas sobre la educación se remonta a la aplicación del concepto de profecía autocumplida a los profesores, según lo cual los profesores interactúan con sus estudiantes de tal manera que se cumplan las expectativas que tienen sobre ellos. Desde entonces, se han estudiado exhaustivamente las expectativas de los profesores hacia los estudiantes en diversos contextos, descuidando el estudio de las expectativas de otras personas significativas (Rubie-Davies et al., 2010).

Los estudios muestran una estrecha relación entre la participación y las expectativas de los padres y madres sobre la educación de sus hijos. Zhan (2006) encontró que aquellos padres que tenían mayores expectativas sobre la educación de sus hijos, presentaban a su vez comportamientos específicos como la participación permanente en actividades escolares y el establecimiento de normas para la escolarización.

Por otra parte, se ha evidenciado una relación positiva entre las expectativas sobre la educación y el rendimiento académico de los niños (Cox, Jamentb y Tarry, 2011; Galindo 
y Sheldon, 2012; Rivera y Milicic, 2006), como también una asociación con el nivel socioeconómico, en donde los padres de estudiantes de nivel socioeconómico más bajo presentarían un escaso interés por la continuación de estudios, ligado a la creencia de que sería una pérdida de tiempo que escasamente influiría en el futuro laboral (Weinberg, 2009), y en sentido inverso, aquellos padres con mayores ingresos económicos esperarían que sus hijos alcancen mayores niveles educativos (Lippman et al., 2008 Cit. en Stull, 2013).

Sin embargo, otros estudios sugieren que las expectativas educativas de los padres serían una variable mediadora entre el nivel socioeconómico y los resultados académicos (Davis-Kean, 2005; Schmitt-Wilson, 2012; Stull, 2013), permitiendo concluir que los padres que tienen altas expectativas educativas para sus hijos, independientemente de la posición social, son más propensos desarrollar en los niños altas expectativas educativas para sí mismos. Así también, las personas de nivel socioeconómico bajo que han aumentado las expectativas educativas son más propensas a tener expectativas laborales similares a las de sus pares de nivel socioeconómico más alto (Schmitt-Wilson, 2012).

Otro estudio presenta una visión distinta del rol de las expectativas educativas de los padres sobre el logro académico de sus hijos, abordando esta variable dentro del concepto de capital cultural escolar, que junto con el nivel socioeconómico configurarían variables mediadoras entre el estatus sociocultural-económico y el logro académico (Hernández y González, 2011).

Ligado a la relación entre expectativas y logro académico, Carro et al. (2014) han identificado las expectativas bajas de los padres junto con el bajo nivel educativo de los padres, el gran número de hermanos y la interrupción familiar como factores de dominio familiar que inciden en la exclusión educativa, particularmente sobre la deserción escolar.

Por otra parte, respecto de la relación entre el estilo parental y las expectativas educativas, Zhan (2006) encontró que los padres con mayores expectativas para sus hijos tienen más probabilidades de establecer normas más estrictas para la escolarización de sus hijos, a diferencia de los padres con expectativas educativas más bajas.

Finalmente, existe evidencia de que la consistencia entre las expectativas educativas de los padres y las expectativas educativas de los hijos favorece el alcance de las metas educativas en los niños (Beutel y Anderson, 2008).

Los antecedentes revisados permiten dilucidar, en primer lugar, que la educación es clave para la integración social y desarrollo de los niños y niñas. Esta función social de la educación es especialmente relevante en contextos de vulnerabilidad socioeconómica, favoreciendo la movilidad social de los estudiantes.

Por otra parte, se ha reconocido que la educabilidad de los niños y niñas se ve afectada por variables del entorno familiar, tales como la participación y las expectativas, donde resulta indispensable fortalecer la organización de la comunidad escolar de tal manera que facilite la participación de todos los actores educativos, entre ellos los padres, madres y apoderados.

En tal sentido, este estudio busca conocer la relación entre la participación y las expectativas de los padres sobre la educación de sus hijos, en el contexto de una escuela municipal de enseñanza básica y enseñanza artística del sur de Chile. Resulta pertinente indagar a su vez en la relación de estas variables con el proceso de aprendizaje de los niños y niñas, y abrir así, de cara al futuro, posibles estrategias de intervención y mejora escolar orientadas a los padres, para hacer de los sueños educativos y profesionales de los estudiantes una realidad.

En definitiva, este estudio intenta dar respuesta a la necesidad del establecimiento de conocer, en general, los aspectos involucrados en la participación y las expectativas de 
los padres y madres sobre la educación de sus hijos; y, adicionalmente, responder a la necesidad científica de ampliar el estudio de la participación y de las expectativas a través de diseños mixtos que permitan hacer inteligibles aquellas condiciones contextuales de los espacios de investigación educativa, alcanzando una comprensión más amplia de los problemas que se presentan en este campo (Creswell, 2012; Pereira, 2011).

Es así que la pregunta que guía la investigación queda definida como: ¿Cuál es la relación entre la participación de los apoderados en la educación, las expectativas sobre el nivel educativo que alcanzarán sus hijos(as) y el rendimiento académico de estos?

\section{MÉTODO}

\subsection{DISEÑO}

Corresponde a un estudio mixto, con un diseño secuencial explicativo, compuesto de una primera fase cuantitativa, no experimental, de alcance explicativo y de temporalidad transversal (Creswell, 2012); seguido de un segundo estudio complementario, basado en los lineamientos de la Teoría Fundamentada (Strauss y Corbin, 2002).

\subsection{PARTICIPANTES}

En la primera fase se intentó trabajar sobre una muestra censal, vale decir, que abarcara todo el universo de apoderados del establecimiento, en los niveles de pre-kínder a octavo básico. Se incluyeron en la muestra a todos aquellos participantes que correspondían al padre, madre, apoderado o adulto responsable inscrito en el registro de matrícula del estudiante. Se entregó un total de 437 cuestionarios, equivalentes al total de participantes presupuestados, de los cuales fueron devueltos 310, siendo eliminados dos casos que correspondían a cuestionarios que se presentaban con omisiones o errores en sus respuestas. Finalmente, la muestra obtenida que se sometió a análisis (308 casos), constituye el 70,80\% del universo estudiado. Todos los sujetos participaron voluntariamente. La muestra presentó una distribución de género de 264 mujeres $(85,7 \%)$ y 44 varones $(14,3 \%)$, mientras que el rango de edad osciló entre los 16 y los 69 años $(M=37.48, D E=9.42)$.

En la segunda fase del estudio se seleccionó a los participantes mediante un muestreo intencional (Martín-Crespo y Salamanca, 2007), logrando la saturación teórica de las categorías conceptuales con nueve participantes. Los participantes fueron seleccionados buscando casos de la tendencia central de la primera muestra, esto es, apoderadas mujeres, en su mayoría adultas jóvenes, lo que responde al propósito de profundizar en la generalidad de los resultados obtenidos en la primera parte del estudio (Creswell, 2012).

\subsection{INTRUMENTOS}

\subsubsection{Fase cuantitativa}

a) Cuestionario sociodemográfico: Este cuestionario recogió las siguientes variables: edad, género, parentesco o relación con el estudiante, nivel socioeconómico percibido tanto de la familia de origen del apoderado como del grupo familiar actual del alumno(a), nivel educativo de la madre y nivel educativo del padre. 
b) Escala de participación de los apoderados en la educación escolar de los hijos: El instrumento fue construido a partir de las distintas conceptualizaciones del constructo de participación en la educación que se han reportado en la literatura, utilizando una escala tipo Likert con cuatro opciones de respuesta que oscilan desde Nunca (1) hasta Siempre (4), compuesto de 22 indicadores agrupados en tres dimensiones: Participación democrática en la escuela, apoyo a la educación desde el hogar e inversión de recursos en educación.

c) Cuestionario de expectativas de los apoderados sobre la educación de sus hijos: Se empleó un cuestionario de dos preguntas orientadas a conocer las expectativas acerca del nivel educativo que los niños(as) alcanzarán y el tipo de profesión en la que se desempeñarán, basado en los antecedentes reportados en contextos norteamericanos, europeo y latinoamericano respecto de la utilización de estas preguntas en la medición de la variable de expectativas sobre la educación (Hernández y González, 2011; Intxausti et al., 2014; Stull, 2013).

\subsubsection{Fase cualitativa}

Entrevista semi-estructurada: Se construyó una pauta de entrevista semi-estructurada a partir de los resultados obtenidos en la primera fase del diseño de estudio, de acuerdo a los lineamientos de Creswell (2012). La entrevista fue organizada en dos ejes temáticos: (1) Participación en la educación, aborda las temáticas del rol del apoderado, las formas de participación y las barreras a la participación, tanto desde el hogar como en relación con la escuela y (2) Expectativas sobre la educación, aborda las temáticas de expectativas educativas y laborales de los padres, formación de expectativas educativas, condiciones que permiten el logro de las expectativas educativas y expectativas de los profesores sobre los alumnos.

\subsection{PROCEDIMIENTO}

En primer lugar se obtuvo la autorización institucional para la realización del estudio, por parte del director del establecimiento. La aplicación de cuestionarios se realizó en reunión normal de apoderados o por envío con el alumno al hogar. Todo esto se realizó durante el desarrollo normal del segundo semestre académico, entregándose junto al cuestionario una carta de consentimiento informado explicando los propósitos y resguardos éticos del estudio. En todos los casos, se respondió el cuestionario de forma individual, apelando a la sinceridad de los apoderados. Una vez recolectados, se realizó un procesamiento computacional de los datos obtenidos a través de las escalas aplicadas, mediante el software IBM SPSS Statistics versión 20.0.

Durante la segunda fase del estudio, las entrevistas fueron realizadas en la sala de entrevistas a apoderados del establecimiento. Cada entrevista fue grabada mediante registro de audio. Previamente a su realización, se hizo entrega y lectura expresa de la carta de consentimiento informado a cada participante, explicando los propósitos y resguardos éticos del estudio. 


\section{RESULTADOS}

\subsection{FASE CUANTITATIVA}

Para evaluar el efecto de las variables recogidas en el estudio sobre la variable rendimiento académico, se llevó a cabo un análisis de regresión lineal múltiple, a través del método de pasos sucesivos (se han omitido los modelos menos relevantes), agrupando las variables de expectativas de los apoderados, nivel socioeconómico y participación de los apoderados como los principales predictores del rendimiento académico de los estudiantes. Dentro de los supuestos que permiten garantizar la validez del modelo de regresión, se encontró independencia en los residuos (Durbin-Watson=1.708) y ausencia de colinealidad entre los predictores (ver Tabla 1).

El resultado del modelo obtenido corresponde a un coeficiente $R 2=.151\left(R 2_{a d j}=.140\right)$, con una significación estadística de $F(1,247)=14.609, p=.000$, lo cual permite sostener que existe una relación lineal estadísticamente significativa entre los predictores agrupados y los puntajes de rendimiento académico.

Tabla 1. Coeficientes, correlaciones semiparciales al cuadrado y estadísticos de colinealidad para los resultados de regresión lineal múltiple sobre los puntajes de rendimiento académico

\begin{tabular}{|c|c|c|c|c|c|c|c|}
\hline \multirow{2}{*}{ Modelo } & \multicolumn{2}{|c|}{$\begin{array}{l}\text { Coeficientes no } \\
\text { estandarizados }\end{array}$} & \multirow{2}{*}{$\begin{array}{c}\begin{array}{c}\text { Coeficientes } \\
\text { estandarizados }\end{array} \\
\beta\end{array}$} & \multirow[b]{2}{*}{$t$} & \multirow[b]{2}{*}{$s r^{2}$} & \multicolumn{2}{|c|}{$\begin{array}{l}\text { Estadísticos de } \\
\text { colinealidad }\end{array}$} \\
\hline & B & E.E. & & & & Tolerancia & FIV \\
\hline (Constante) & 4.141 & .286 & & 14.499 & .000 & & \\
\hline Expectativas & .070 & .020 & .218 & 3.569 & .043 & .921 & 1.085 \\
\hline NSE Total & .041 & .014 & .180 & 2.892 & .028 & .888 & 1.126 \\
\hline Participación & .011 & .004 & .170 & 2.825 & .028 & .953 & 1.049 \\
\hline
\end{tabular}

Del análisis de la Tabla 1 se puede concluir que los predictores son estadísticamente significativos en su contribución a la explicación de los puntajes de rendimiento académico. El primero de estos corresponde a las expectativas de los apoderados sobre la educación de sus hijos $(\beta=.218, s r 2=.043)$, seguida del nivel socioeconómico $(\beta=.180, s r 2=.028)$ y de la participación de los apoderados en la educación escolar $(\beta=.170, s r 2=.028)$.

Para determinar la relación entre la participación de los apoderados en la educación respecto del rendimiento académico de sus hijos(as) se realizó un análisis de correlaciones bivariadas empleando el estadístico coeficiente de correlación de Pearson, encontrando que existe una relación estadísticamente significativa entre estas dos variables $(r=.223$, $p=.000$ ). Por otra parte, al analizar la relación entre las expectativas de los apoderados sobre la educación, respecto del rendimiento académico de sus hijos(as), se encontró también una relación positiva y estadísticamente significativa $(r=.283 ; p=.000)$, tal como se muestra en la Tabla 2. 
Estudios Pedagógicos XLII, N 3: 347-367, 2016

PARTICIPACIOON Y EXPECTATIVAS DE LOS PADRES SOBRE LA EDUCACIÓN DE SUS HIJOS EN UNA ESCUELA PÚBLICA

Tabla 2. Correlaciones entre las variables centrales del estudio

\begin{tabular}{|c|c|c|c|c|c|c|c|c|}
\hline & 1 & 2 & 3 & 4 & 5 & 6 & 7 & 8 \\
\hline 1. Edad del apoderado & 1 & .023 & $.129^{*}$ & .065 & $.134^{*}$ & $.127^{*}$ & -.042 & $-.141^{*}$ \\
\hline 2. Rendimiento académico & & 1 & $.139 *$ & $.201^{* * *}$ & $.219^{* *}$ & $.223^{* *}$ & $.271^{* *}$ & $.283^{* *}$ \\
\hline 3. Participación democrática & & & 1 & $.549^{* *}$ & $.488^{* *}$ & $.854^{* *}$ & $.130^{*}$ & -.036 \\
\hline 4. Apoyo desde el hogar & & & & 1 & $.477^{* * *}$ & $.858^{* *}$ & $.167^{* * *}$ & $.122^{*}$ \\
\hline 5. Comunicación con la escuela & & & & & 1 & $.727^{* *}$ & $.167^{* * *}$ & .100 \\
\hline 6. Participación en la educación & & & & & & 1 & $.185^{* *}$ & .069 \\
\hline 7. Nivel socioeconómico & & & & & & & 1 & $.314^{* *}$ \\
\hline 8. Expectativas educativas & & & & & & & & 1 \\
\hline
\end{tabular}

*. La correlación es significativa al nivel .05 (bilateral).

**. La correlación es significativa al nivel .01 (bilateral).

Adicionalmente, los resultados muestran una relación positiva entre el nivel socioeconómico y el rendimiento académico de los estudiantes $(r=.271, p=.000)$, como también con las expectativas de los apoderados sobre la educación $(r=.314, p=.000)$.

Para evaluar el efecto mediador de las expectativas de los apoderados sobre la educación, entre el rendimiento académico y el nivel socioeconómico, se realizó adicionalmente un análisis de correlaciones semi-parciales, encontrándose una relación más baja que la obtenida sin controlar por las expectativas $(r=.211, p=.000)$.

\subsection{FASE CUALITATIVA}

\subsubsection{Resultados de la codificación abierta}

En el análisis descriptivo de las entrevistas realizadas emergieron dos categorías centrales en el estudio, que son la participación de los apoderados en la educación y las expectativas de los apoderados acerca del nivel de educación que alcanzarán sus hijos.

Respecto de la participación de los padres, madres y apoderados en la educación de sus hijos, se encontró, en primer lugar, que el rol de los apoderados puede distinguirse según si está asociado al alumno o a la escuela y los profesores. En el primer caso, consistiría en apoyar al alumno, informarse de su actividad diaria, estar pendiente de su estudio, motivarlo e incentivarlo para que estudie, acompañarlo cuando se requiera en la escuela y proveerle de transporte para asistir a clases. En relación con rol del apoderado para con la escuela y los profesores, este abarcaría el asistir a las reuniones de apoderados, estar en comunicación con la escuela, alinearse al proyecto educativo institucional, informarse sobre lo que ocurre en la escuela y apoyar a los profesores.

Otra categoría emergente en el análisis descriptivo fue la importancia que tiene la participación de los apoderados en la educación, incluyéndose dentro de esta categoría el hecho de permitir estar pendiente del avance del alumno, pudiendo expresarse este avance 
en términos de rendimiento escolar o comportamiento. Además, dentro de las razones para participar estarían la obtención de beneficios para el alumno, la posibilidad de resolver problemas del alumno y del curso y el hecho de que la participación facilitaría el logro de expectativas educativas. Un ejemplo de ello es lo declarado en la siguiente entrevista:

“[...] uno tiene que saber lo que el niño está aprendiendo para también practicarlo en la casa, uno tiene que saber lo que está haciendo el colegio, lo que dicen los niños, lo que pasa todos los días, porque conozco casos no muy lejanos que los papás no tienen idea de la materia que van, no tienen idea cuándo tienen prueba, no tienen idea de los materiales que hay que traer y uno tiene que andarles diciendo o andan preguntando". (Apoderada adulto joven, III, 3).

Dentro de las formas de participación señaladas por las apoderadas se distinguieron dos dimensiones de participación: el hogar y la escuela. Dentro de la participación en el hogar se incluiría el ayudar y supervisar realización de tareas, estudiar las materias, formar hábitos de responsabilidad y respeto, revisar cuadernos, brindar atención y cariño al alumno y proporcionar medios para realizar tareas y trabajos.

Por otra parte, dentro de las formas de participar en la escuela se incluirían, en primer lugar, las reuniones de apoderados, que se caracterizarían por ser frecuentes y obligatorias, permitiendo recibir información del curso en general, informarse sobre las notas, registrar el avance o retroceso del alumno, conocer si hay tareas para la casa, recibir información de la escuela, resolver dudas sobre los contenidos vistos en clase con el profesor, interactuar con otros apoderados y recibir información del Centro General de Padres y Apoderados:

“... ahi escuchamos las notas que tienen los hijos y si van atrasados, si estudian, entonces uno se pone al día en la reunión con todo y ahí uno sabe al tiro si mandaron tarea para la casa o no, entonces de repente dicen que no y mandaron tarea". (Apoderada adulto medio, I, 3).

Finalmente, respecto a las formas de participación, se destacan formas de participación informativa, tales como los días de atención de los profesores a los apoderados, las llamadas por teléfono al profesor, el contacto con profesionales del establecimiento, la comunicación por escrito con el profesor, el asistir a la escuela a preguntar por las notas y la información a través de redes sociales.

Dentro de las barreras a la participación, se distinguen las barreras desde el hogar y desde la escuela. En las primeras se incluiría el trabajo de los padres, la deficiencia en competencias parentales para tratar con el niño en el hogar, la escasez de recursos económicos y problemas de salud en el grupo familiar. En las segundas se señala el desinterés de los otros padres, la falta de cohesión de los apoderados y la débil gestión del Centro General de Padres y Apoderados. Un ejemplo de la barrera que presenta mayor recurrencia en los datos es el trabajo de los apoderados:

“...desde que estoy trabajando, la verdad que no me puedo acercar mucho al colegio, incluso ahora estoy con licencia y por eso he podido hacer varios trámites, pero la verdad que ya hace como un año que no soy muy cercana al colegio, pero sí a las reuniones, si no puedo venir yo vienen los abuelos, pero siempre ahí pendiente". (Apoderada adulto joven, VIII, 4).

Finalmente, como consecuencias a la falta de participación de los apoderados se reconocen la desinformación respecto del curso y la escuela y la vergüenza que experimentan 
los alumnos frente a sus compañeros y profesores cuando, por ejemplo, no asisten con los materiales necesarios para realizar las tereas.

En relación con las expectativas de los padres, madres y apoderados sobre la educación de sus hijos, se encontró que las expectativas educativas pueden corresponder a un nivel de estudios universitarios o al nivel de estudios técnico-profesionales. Se encontró también que la formación de estas expectativas educativas estaría dada por modelos familiares, las expectativas de los profesores y las expectativas sociales.

Dentro de las condiciones para el logro de las expectativas, se reconoció el esfuerzo de los apoderados y la familia, la dedicación única del alumno a estudiar, los recursos económicos para el financiamiento, la calidad de la educación recibida, la calidad de los profesores y la relación entre la escuela y la familia.

“...una carrera técnica, porque, porque si bien están las becas y todo yo sé que a lo mejor mi hija puede más, puede llegar a la universidad, pero los medios no están, entonces ¿hasta dónde puedo optar yo? hasta una carrera técnica, nada más, pero por mí mucho más, yo sé que mi hija da mucho más, pero por el tema de los recursos yo podría optar hasta ahí”. (Apoderada adulto joven, II, 19).

Por otra parte, sobre las expectativas de los profesores se encontró que pueden ser altas o desconocidas. En el primer caso se reconoció que los profesores con altas expectativas suelen reforzarlas grupalmente, y son altas respecto de aquellos alumnos con buen rendimiento académico, con un buen comportamiento y con características familiares favorables para su educación.

Finalmente, respecto de las expectativas laborales, se señala solo el hecho de tener una profesión, además de las aspiraciones de los padres acerca de que el alumno sea más que el apoderado y que lo que alcancen en términos educativos sea lo mejor para ellos.

\subsubsection{Resultados de la codificación axial}

A continuación se presenta el producto de la codificación axial de los dos ejes temáticos del estudio, explicitando el tipo de relaciones que se da entre las distintas categorías emergentes en el primer análisis.

En primer lugar, se presentan los resultados en relación con el eje de participación de los apoderados en la educación escolar de sus hijos, que está sintetizado en la Figura 1.

Es posible visualizar que las distintas condiciones que intervienen en el fenómeno de la participación de los apoderados están presentes en dos dimensiones básicas: el hogar y la escuela, que modulan tanto las formas de participación y las barreras que las obstaculizan; todo esto dentro de un contexto espacial, temporal, social, económico y cultural propio de la dinámica estudiada. 
Figura 1. Paradigma de codificación:

Participación de los apoderados en la educación de sus hijos

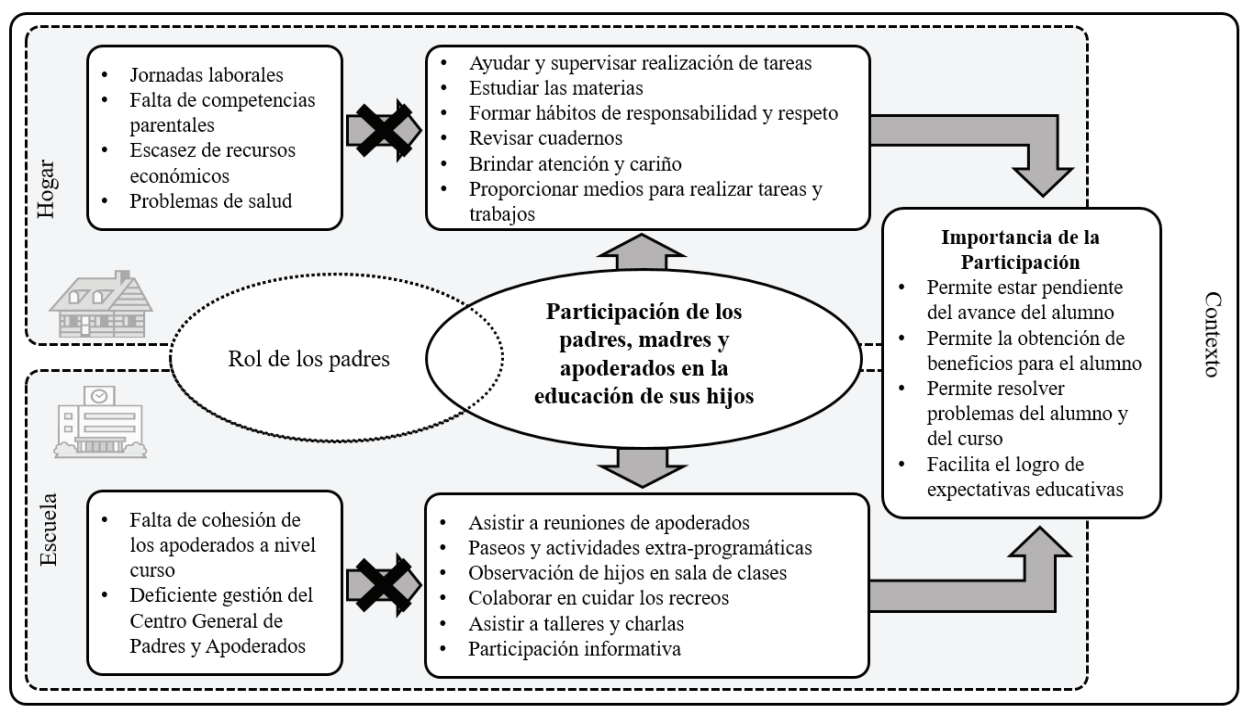

Los resultados muestran que el rol de los padres y apoderados se cruza con elementos de lo que significa participar en la educación escolar, no siendo todas las funciones de un apoderado parte de la participación, ni todas las formas de participar en la educación una función del apoderado. Es decir, correspondería a la intersección de dos conjuntos independientes. Entre los elementos comunes del rol del apoderado que constituyen formas de participación se encontrarían, en relación con el alumno, la función de apoyarlo y acompañarlo en sus tareas; y en relación con la escuela, el asistir a las reuniones de apoderados e informarse de lo que acontece en ella. Por lo tanto, desde el discurso de las apoderadas entrevistadas, estas serían funciones elementales que todo apoderado debe cumplir, como un primer eslabón de la participación.

Respecto de las barreras a la participación, existe consenso entre las apoderadas en que desde la escuela la principal barrera estaría dada por el conjunto de apoderados y su organización como estamento a través del Centro General de Padres y Apoderados.

Asimismo, es posible notar que existe una relación entre la principal barrera presente desde el hogar, que son las jornadas de trabajo, y la barrera desde la escuela, que es la falta de cohesión de los apoderados. Cabe notar también que dentro de las razones que justifican la importancia de la participación de los apoderados se señala el hecho de que facilitaría el logro de las expectativas educativas, encontrando así una relación con el segundo eje temático de esta parte del análisis.

Al igual que la participación, las expectativas educativas que los apoderados tienen sobre sus hijos estarían moldeadas por condiciones del contexto social, del hogar y de la escuela (ver Figura 2).

En primer lugar, los modelos familiares que los niños y niñas tienen en sus hogares serían un factor que incide en la formación de expectativas de los padres sobre sus hijos, 
sirviendo como ejemplos que guían los intereses de los niños y niñas en su futura elección vocacional: “... a lo mejor uno no le va a decir: tení’ que estudiar esto, pero si él vio a su tata toda la vida trabajar en esto, siendo profesor y siendo mecánico, a lo mejor le gusta” (Apoderada adulto joven, III, 17).

Un segundo factor que desde la escuela incide en la formación de las expectativas educativas serían las expectativas de los profesores. Esto quiere decir que en la medida en que el profesor refuerce al apoderado ciertas expectativas sobre el nivel de educación que alcanzará el alumno, el apoderado tenderá a coincidir o estructurar su expectativa según las referencias del profesor.

Figura 2. Paradigma de codificación:

Expectativas de los apoderados sobre la educación de sus hijos

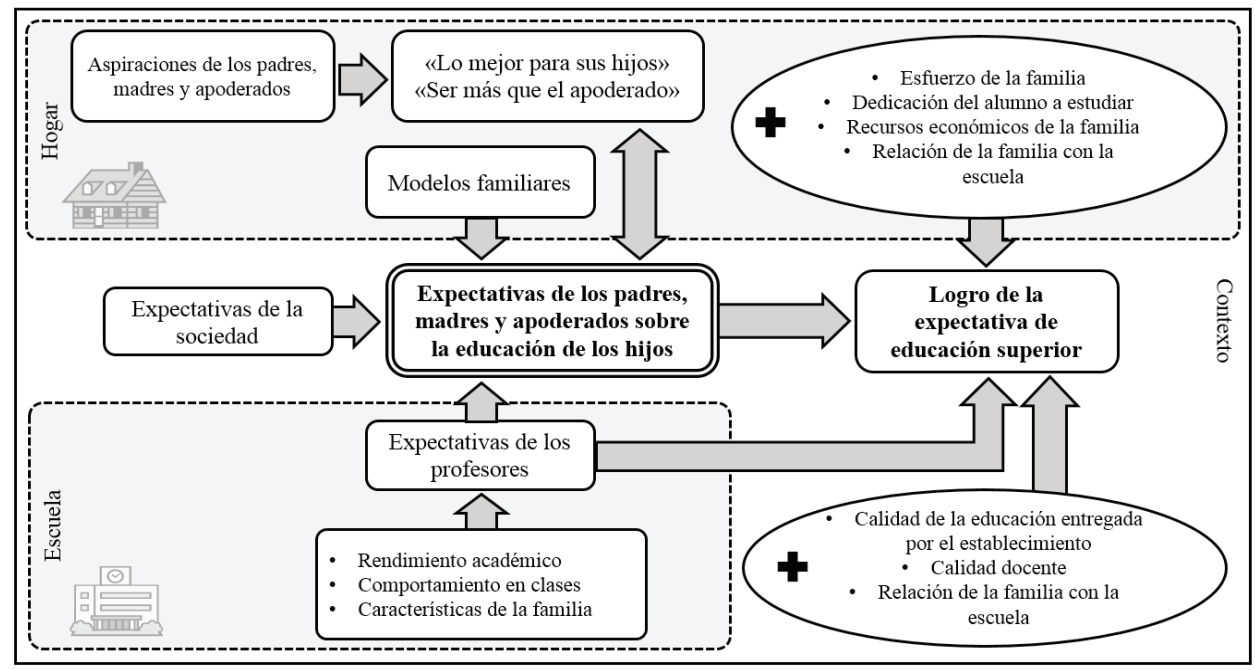

Al profundizar acerca de la percepción de las apoderadas sobre las expectativas de los profesores, se encontró que en su mayoría corresponden a altas expectativas sobre sus estudiantes, que suelen reforzarse grupalmente en las reuniones de apoderados. A pesar de ser altas expectativas, cabe señalar que están mediadas por el rendimiento académico del alumno, el comportamiento en clases y el conocimiento que el profesor tiene sobre la familia del niño. Por lo tanto, las expectativas más altas de los profesores estarán dirigidas hacia aquellos estudiantes con un rendimiento académico alto, con un buen comportamiento y con características de la familia que, a juicio del profesor, facilitarían el logro de dicha expectativa; aunque hay excepciones de profesores que reflejan altas expectativas de sus alumnos a pesar de condiciones desfavorables del entorno familiar. Un ejemplo de lo anterior es el referido por estas apoderadas en relación con las expectativas educativas que los profesores tienen de sus hijos: "Tengo muy buenas referencias de los dos que tengo acá, los dos profesores me felicitan por la enseñanza, por el comportamiento que tienen acá en el colegio, han sacado buenas notas, los primeros lugares y buen rendimiento, 
comportamiento, así que tengo buenas referencias de ellos al menos" (Apoderada adulto joven, VIII, 10).

Por último, un tercer factor que incidiría en la formación de las expectativas educativas de los apoderados serían las expectativas sociales acerca del nivel de educación que es esperable que los niños y niñas alcancen.

Respecto de las condiciones que inciden positivamente en el logro de una expectativa educativa, las apoderadas refieren principalmente —en la dimensión del hogar-, el esfuerzo de los apoderados y la familia, la dedicación del alumno a estudiar y los recursos económicos para el financiamiento. Nuevamente esto refleja la relación de las expectativas con la participación, siendo la participación de los apoderados desde el hogar, mediado por los recursos económicos, condiciones necesarias para que los estudiantes alcancen cierto nivel de educación.

Finalmente, desde la dimensión escuela, las apoderadas señalan que para lograr las expectativas sobre la educación de sus hijos es también necesario que reciban una educación de calidad, que los profesores que la impartan sean de excelencia, y que exista una comunicación permanente entre la familia y la escuela, como lo destaca esta participante: "Depende [...] fundamentalmente de los papás y la escuela, y de la comunicación entre los dos. La relación entre la escuela y la familia, si no se apoyan los dos el niño no va a salir adelante" (Apoderada adulto joven, IV, 11).

\subsection{INTEGRACIÓN DE RESULTADOS}

Los resultados de la fase cualitativa del estudio permiten comprender con mayor profundidad la relación entre las variables centrales del estudio, las variables intervinientes y las dimensiones y contextos en que se expresa ese conjunto de variables.

La Figura 3 resalta en primer lugar la relación entre las variables centrales del estudio; son la participación de los apoderados, el nivel socioeconómico y las expectativas sobre la educación los principales predictores del rendimiento académico del estudiante.

El análisis integrado de la variable participación de los apoderados en la educación permite identificar, a partir de la segunda fase del estudio, algunas formas que reviste la participación en el contexto del establecimiento, según las tres dimensiones del constructo confirmadas en la primera fase. Así, para el caso del apoyo de la educación desde el hogar, algunas formas corresponderían a la supervisión en la realización de tareas, estudio de materias y formación de hábitos de responsabilidad y respeto.

En el caso de la comunicación con la escuela, se presentarían formas como asistir a reuniones y citaciones de apoderados, llamar por teléfono al profesor o al establecimiento y comunicación a través de redes sociales, lo que estaría a su vez asociado a formas de participación informativa.

En tercer lugar, respecto a la participación democrática, los resultados de la segunda fase no permiten profundizar más allá de un nivel informativo, lo que estaría relacionado con una falta de cohesión de los apoderados a nivel de curso y establecimiento (a través del Centro General de Padres y Apoderados). Este hecho en sí mismo constituiría una barrera a otras formas de participación dentro de la escuela.

Adicionalmente, las tres dimensiones de la participación estarían relacionadas con el rol del apoderado, esto es, aquellas funciones que deben cumplir necesariamente por el hecho de ser padres, madres o adultos responsables del proceso escolar de los estudiantes. 
Otra variable asociada a la participación sería la edad del apoderado, siendo mayor la participación en apoderados de mayor edad. Por otra parte, el trabajo de los apoderados y el nivel socioeconómico se constituirían como barreras a la participación.

Figura 3. Análisis selectivo: Integración de datos cuantitativos y cualitativos del estudio

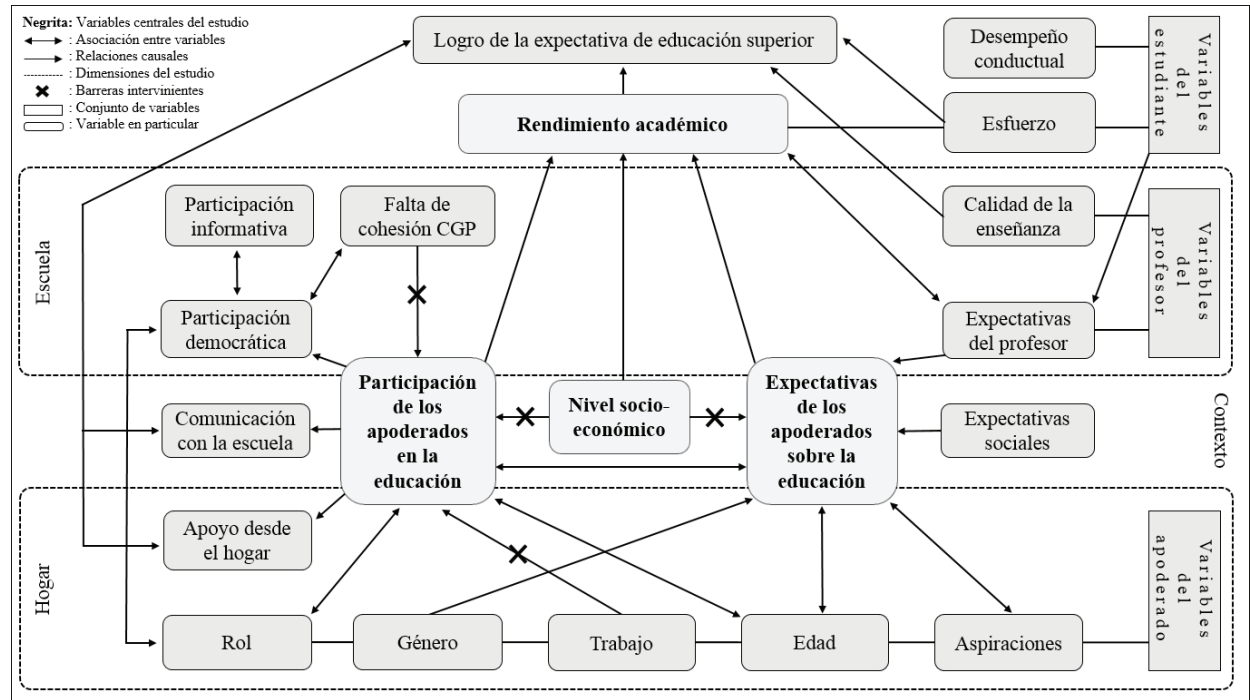

Por otra parte, dos de las dimensiones de la participación, a saber, la comunicación con la escuela y el apoyo desde el hogar, junto con el esfuerzo del estudiante, la calidad de la enseñanza del profesor y el nivel socioeconómico, serían factores que influyen directamente en el logro de las expectativas educativas.

En relación con el análisis de las expectativas sobre la educación, se puede señalar que estarían explicadas por las expectativas del profesor, las expectativas de la sociedad, el nivel socio-económico y el género del apoderado (siendo más altas en apoderados hombres). A su vez, estas expectativas estarían asociadas positivamente a las aspiraciones y a la participación de los apoderados, mientras que tendrían una relación negativa con la edad (siendo más bajas a mayor edad).

La principal barrera encontrada en la formación de expectativas educativas es el nivel socioeconómico del alumno y su familia, lo que constituye una barrera común con la variable participación. Es decir, que los apoderados con menos recursos tendrán más bajas expectativas sobre el nivel de educación que alcanzarán sus hijos, lo que se asociaría a un menor compromiso con el proceso educativo de los estudiantes.

Finalmente, se encontró que las variables del estudiante como el desempeño académico y conductual serían predictoras de las expectativas de los profesores sobre la educación de sus alumnos. 


\section{DISCUSIÓN Y CONCLUSIONES}

Los hallazgos de este estudio permiten sostener que tanto la participación de los apoderados en la educación escolar de sus hijos como las expectativas que tienen acerca del nivel de educación que estos alcanzarán se relacionan positivamente con el rendimiento académico de los niños.

Respecto de la participación de los apoderados, los resultados amplían los antecedentes presentados por Altschul (2012) y Valdés et al. (2013) acerca de la estructura bidimensional de este constructo, añadiéndose al apoyo desde el hogar y la participación en la escuela una dimensión relacional o comunicativa entre ambos escenarios de participación, lo que a su vez se mostró que está asociado a formas de participación informativa, limitadas a la recepción de información acerca del proceso educativo de los estudiantes. Este resultado es coherente con el derecho que tienen los apoderados a ser informados del proceso educativo, expresado en la Ley General de Educación (Ministerio de Educación, 2009), no obstante, los antecedentes encontrados no permiten asegurar que se promueva de forma efectiva la participación de los apoderados a través del centro general y los microcentros. Por lo anterior, es posible pensar que los apoderados de la escuela no cuentan con instancias en que puedan ser consultados en los procesos de toma de decisiones institucionales, participar representativamente en dichos procesos, supervisar la rendición de cuentas sobre los resultados de aprendizaje, evaluar dichos resultados o participar de los procesos de enseñanza; de acuerdo a lo que los autores reconocen como el carácter democrático de la participación; lo que resulta relevante al considerar que esta forma de participar en la educación escolar está asociada a mejores resultados educativos (Gatt y Petreñas, 2012; Valdés y Urías, 2010; Valdés et al., 2013).

En relación con las expectativas de los apoderados sobre la educación de sus hijos y su relación con la variable participación, los resultados confirman lo encontrado en el estudio de Zhan (2006), donde aquellos padres que tenían mayores expectativas sobre la educación de sus hijos presentaban, a su vez, una alta participación en actividades escolares y un mayor apoyo al proceso educativo desde el hogar. Asimismo, este estudio confirma la relación positiva entre las expectativas sobre la educación y el rendimiento académico de los niños reportada en la literatura, incluyendo los estudios en contextos chilenos similares (Cox et al., 2011; Galindo y Sheldon, 2012; Rivera y Milicic, 2006).

Sobre el objetivo de indagar en las condiciones que en la escuela y en el hogar favorecen u obstaculizan una alta participación de los apoderados, se encontró que desde la escuela la principal barrera sería la falta de cohesión del estamento, tanto a nivel del Centro General de Padres y Apoderados como a nivel de microcentros. Por otra parte, desde el hogar la principal barrera sería el trabajo de los apoderados con jornadas laborales que les impide participar, inclusive, de actividades de carácter obligatorio, como las reuniones de apoderados o bien apoyar a los niños desde el hogar.

Estos resultados coinciden con las barreras a la participación encontradas en el estudio de Anabalón et al. (2008), donde identificaron las jornadas de trabajo extensas dentro de los principales factores que limitan la participación de los apoderados en la escuela, junto con el nivel educacional de los padres, no obstante este último factor no mostró una relación significativa con la participación en la primera fase de la investigación.

A su vez, estos resultados pueden ser interpretados a la luz de lo encontrado por Rivera y Milicic (2006) en el contexto chileno, pudiendo asociar a los apoderados del 
establecimiento con el grupo menos participativo de dicho estudio, caracterizado precisamente por apoderados que no asisten por razones de trabajo o desinterés por la educación de sus hijos.

Con todo, esto es discutible al revisar los resultados de un estudio más reciente (Román, 2014) en las escuelas municipales chilenas, donde se encontró que la participación y compromiso de los apoderados se ha reducido en los últimos años, fundamentalmente debido a los cambios en la estructura familiar, compuesta en su mayoría de apoderadas mujeres y trabajadoras, lo que coincide en parte con las características de la población de estudio. De ser así, el desinterés de los apoderados por la educación de sus hijos pasaría a un segundo plano a la hora de explicar la escasa participación.

Por otra parte, respecto de la pregunta acerca de las condiciones que modulan las expectativas educativas, se encontró que el principal determinante en la escuela es el rendimiento académico del alumno y su comportamiento en clases, mediados también por las expectativas del profesor. Desde el hogar, en cambio, la principal condición que determina las expectativas que los apoderados se crean sobre el nivel de educación que alcanzarán sus hijos es el nivel socioeconómico, asociado a la posibilidad de financiar los estudios de educación superior.

Esta última barrera encontrada sería consistente con lo encontrado por Weinberg (2009), en donde los padres de estudiantes de nivel socioeconómico más bajo presentaban un menor interés por la continuación de estudios, ligado a la creencia de que sería una pérdida de tiempo que escasamente influiría en el futuro laboral, mientras que aquellos apoderados con mayores ingresos económicos esperarían mayores niveles educativos de sus hijos.

Otro hallazgo en relación con las expectativas y coincidente con la literatura (DavisKean, 2005; Schmitt-Wilson, 2012; Stull, 2013) es el hecho de que las expectativas de los padres pueden mediar la relación existente entre el nivel socioeconómico y los resultados académicos. Así, los apoderados que tengan altas expectativas sobre sus hijos con independencia de las condiciones de su medio social y económico, es posible que logren mejores resultados educativos en los niños que aquellos padres que sostengan bajas expectativas.

En definitiva, los resultados de este estudio tienden a confirmar lo reportado en la literatura en torno a las variables de participación y expectativas de los apoderados. Dentro de las contribuciones del estudio se puede señalar un claro aporte al estado del arte del tema en el contexto chileno, especialmente en relación con las expectativas de los apoderados sobre la educación, ya que los estudios revisados tienden a poner el foco en las expectativas de los profesores, descuidando la incidencia que pueden tener otras personas significativas para los estudiantes (Rubie-Davies et al., 2010).

Otra contribución del estudio en términos metodológicos es aportar a la investigación educativa a través de diseños mixtos que permitan evidenciar aquellos procesos del contexto social y cultural de la investigación, alcanzando una comprensión más amplia de los problemas que se presentan en los establecimientos, y generando, a partir de ese conocimiento, nuevas propuestas para la mejora educativa (Creswell, 2012; Pereira, 2011).

Desde estos resultados se presenta el desafío de desarrollar estrategias dentro de la escuela para fortalecer las expectativas que los apoderados y los profesores tienen sobre los estudiantes, lo que resulta especialmente relevante en contextos educativos marcados por altos niveles de vulnerabilidad social y económica, que tienden a condicionar bajas expectativas sobre el desarrollo de los alumnos, atribuidas al entorno social y familiar del que provienen. 
Adicionalmente, se destaca el desafío de hacer frente a las barreras que desde el hogar obstaculizan la participación de los apoderados: cómo diseñar espacios de participación compatibles con las jornadas de trabajo o qué puede hacer la escuela frente al eventual desinterés de los padres por acompañar a sus hijos en su proceso educativo.

Un análisis de las deficiencias del estudio permite relevar ciertos aspectos metodológicos, tanto de la primera como de la segunda fase del estudio. En la fase cuantitativa, se puede señalar el uso de escalas de autorreporte, es sabido que son mayormente susceptibles de ser respondidas por la deseabilidad social. Otro aspecto de esta fase tiene que ver con la debilidad de las conclusiones que se pueden arrojar desde los resultados obtenidos en los análisis de correlaciones, donde si bien se presenta una serie de relaciones entre las variables que confirman lo reportado en la literatura, en general tienen un escaso valor predictivo (tratándose del análisis de regresión lineal múltiple) y una baja asociación estadística en el caso de las correlaciones $(r<.4)$.

Entre los límites de la segunda fase del estudio, cabe señalar la posibilidad de haber realizado un muestreo de máxima variabilidad para indagar en los distintos perfiles de apoderados del establecimiento, abriendo la posibilidad de encontrar resultados distintos en relación con las formas de participación y las barreras que se presentan desde la realidad de cada uno de ellos.

Adicionalmente, habría sido posible enriquecer los resultados a través de la realización de más entrevistas que abordaran nuevos tópicos emergentes, tales como las expectativas de los profesores o la importancia de la participación democrática en la escuela, que fueron en menor medida abordadas, fundamentalmente por el tiempo disponible para la investigación.

La investigación educativa constituye una estrategia de cambio y mejora educativa, siendo importante como parte de los esfuerzos para fortalecer la educación pública, gratuita y de calidad. Se sugiere para estudios futuros en esta línea indagar en nuevas formas de participación que respondan a los cambios y desafíos que experimenta la sociedad a nivel institucional y de las familias, en línea con paradigmas de educación que integren a los apoderados como agentes activos en el proceso de aprendizaje de los niños y niñas.

Sería también interesante indagar en el impacto que tienen la participación y las expectativas de los apoderados más allá del rendimiento académico de los alumnos, abordando aspectos relativos a la formación integral de ciudadanos, que es uno de los objetivos que se propone la educación en el contexto político chileno.

Es posible concluir así que la educación sigue siendo, para la mayoría de la población, el principal vehículo de movilidad social (Bellei et al., 2014). Esta investigación se suma a la basta evidencia acerca de la influencia que ejercen las condiciones socioeconómicas de las familias sobre el desarrollo de los estudiantes, y se adhiere a la convicción de que esa influencia puede ser revertida o atenuada por la acción que puede llevar a cabo la escuela.

\section{REFERENCIAS BIBLIOGRÁFICAS}

Altschul, I. (2012). Linking socioeconomic status to the academic achievement of mexican american youth through parent involvement in education. Journal of the Society for Social Work and Research, 3(1), 13-30. doi:10.5243/jsswr.2012.2

Anabalón, M., Carrasco, S., Díaz, D., Gallardo, C., \& Cárcamo, H. (2008). El compromiso familiar 
frente al desempeño escolar de niños y niñas de educación general básica en la ciudad de Chillán. Horizontes Educacionales, 13(1), 11-21.

Bellei, C., Valenzuela, J. P., Vanni, X., \& Contreras, D. (2014). Lo aprendí en la escuela: ¿Cómo se logran procesos de mejoramiento escolar? Santiago de Chile: Universidad de Chile.

Beutel, A., \& Anderson, K. (2008). Race and the educational expectations of parents and children: The case of South Africa. The Sociological Quarterly, 49(2), 335-361.

Bolívar, A. (2006). Familia y escuela: dos mundos llamados a trabajar en común. Revista de Educación, (339), 119-146.

Carro, A., Lima, J.A., Hernández, F., \& León, A. (2014). Educar sin excluir. Una experiencia de educación inclusiva en el estado de Tlaxcala, México. Revista Nacional e Internacional de Educación Inclusiva, (1), 140-162.

Cornejo, R. (2006). El experimento educativo chileno 20 años después: Una mirada crítica a los logros y falencias del sistema escolar. Revista Electrónica Iberoamericana sobre Calidad, Eficacia y Cambio en Educación, 4(1), 118-129.

Cox, A., Jamentb, J., \& Tarry, E. (2011). An investigation into parental expectations of primary schooling and the support provided by schools to a single Keralite community in an English county. Procedia Social and Behavioral Sciences, 15, 66-71. doi:10.1016/j.sbspro.2011.03.052

Creswell, J. (2012). Educational research: Planning, conducting, and evaluating quantitative and qualitative research (4th ed.). Boston, MA: Pearson Education.

Davis-Kean, P. (2005). The influence of parent education and family income on child achievement: The indirect role of parental expectations and the home environment. Journal of Family Psychology, 19(2), 294-304. doi:10.1037/0893-3200.19.2.294

Galindo, C., \& Sheldon, S. (2012). School and home connections and children's kindergarten achievement: The mediating effects of family involvement'. Early Childhood Research Quarterly, 27(1), 90-103. doi:10.1016/j.ecresq.2011.05.004

Gatt, S., \& Petreñas, C. (2012). Formas de participación y éxito educativo. Cuadernos de Pedagogía, (429), 50-52.

Hernández, E., \& González, M. (2011). Modelo de ecuación estructural que evalúa las relaciones entre el estatus cultural y económico del estudiante y el logro educativo. Revista Electrónica de Investigación Educativa, 13(2), 188-203.

Intxausti, N., Etxeberria, F., \& Joaristi, L. (2014). ¿Coinciden las expectativas escolares de la familia y del profesorado acerca del alumnado de origen inmigrante? Relieve, 20(1), 1-21. doi:10.7203/ relieve.20.1.3804

Martín-Crespo, M. A., \& Salamanca, A. B. (2007). El muestreo en la investigación cualitativa. Nure Investigación, 27. Recuperado desde http://www.nureinvestigacion.es/OJS/index.php/nure/ article/view/340/330

Martínez, B., \& Niemelä, N. (2010). Formas de implicación de las familias y de la comunidad hacia el éxito educativo. Revista Educación y Pedagogía, 22(56), 69-77.

Ministerio de Educación. (2009). Ley General de Educación. Biblioteca del Congreso Nacional de Chile, Santiago de Chile.

Moreno-Doña, A. y Gamboa, R. (2014). Dictadura chilena y sistema escolar: "A otros dieron de verdad esa cosa llamada educación". Educar em Revista, 51, 51-66. doi:10.1590/S010440602014000100005

Pereira, Z. (2011). Los diseños de método mixto en la investigación en educación: Una experiencia concreta. Revista Electrónica Educare, 15(1), 15-29.

Rivera, M., \& Milicic, N. (2006). Alianza familia-escuela: Percepciones, creencias, expectativas y aspiraciones de padres y profesores de enseñanza general básica. Psykhe, 15(1), 119-135.

Román, M. (2014). Elección y permanencia en escuelas de baja calidad en un sistema educativo de cuasi-mercado. ¿Opción o resignación? (Tesis doctoral). Universidad Autónoma de Madrid.

Rubie-Davies, M., Peterson, E., Irving, E., Widdowson, D., \& Dixon, R. (2010). Expectations of 
achievement. Student, teacher and parent perceptions. Research in Education, 83, 36-53.

Schmitt-Wilson, S. (2012). Social class and expectations of rural adolescents: The role of parental expectations. The Career Development Quarterly, 61, 226-239. doi:10.1002/j.21610045.2013.00051.x

Silas, J. (2008). “Por qué Miriam sí va a la escuela?”. Revista Mexicana de Investigación Educativa, 13(39), 1255-1279.

Strauss, A., \& Corbin, J. (2002). Bases de la investigación cualitativa. Técnicas y procedimientos para desarrollar la teoría fundamentada. Medellín, Colombia: Universidad de Antioquia.

Stull, J. (2013). Family socioeconomic status, parent expectations, and a child's achievement. Research in Education, 90, 53-67. doi:10.7227/RIE.90.1.4

Vain, P. D. (2009). Escuela, Estado y familia. Un pacto por redefinir. Educación, Lenguaje y Sociedad, 6(6), 329-344.

Valdés, A., \& Urías, M. (2010). Creencias de padres y madres acerca de la participación en la educación de sus hijos. Perfiles Educativos, 33(134), 99-114.

Valdés, A., Carlos, E., \& Arreola, C. (2013). Desarrollo de un instrumento para medir la participación de los padres en la educación escolar de los hijos. Revista de Evaluación Educativa, 2(1), $92-$ 109.

Valdés, A., Martín, M., \& Sánchez-Escobedo, P. A. (2009). Participación de los padres de alumnos de educación primaria en las actividades académicas de sus hijos. Revista Electrónica de Investigación Educativa, 11(1), 1-17.

Weinberg, L. (2009). Parents' educational expectations for their young children: SES, racelethnicity and school feedback (Doctoral thesis). The Florida State University.

Zhan, M. (2006). Assets, parental expectations and involvement, and children's educational performance. Children and Youth Services Review, 28, 961-975. doi:10.1016/j. childyouth.2005.10.008 
